\title{
The role of microglia in viral encephalitis: a review
}

\author{
Zhuangzhuang Chen * D, Di Zhong and Guozhong Li
}

\begin{abstract}
Viral encephalitis is still very prominent around the world, and traditional antiviral therapies still have shortcomings. Some patients cannot get effective relief or suffer from serious sequelae. At present, people are studying the role of the innate immune system in viral encephalitis. Microglia, as resident cells of the central nervous system (CNS), can respond quickly to various CNS injuries including trauma, ischemia, and infection and maintain the homeostasis of CNS, but this response is not always good; sometimes, it will exacerbate damage. Studies have shown that microglia also act as a double-edged sword during viral encephalitis. On the one hand, microglia can sense ATP signals through the purinergic receptor P2Y12 and are recruited around infected neurons to exert phagocytic activity. Microglia can exert a direct antiviral effect by producing type 1 interferon (IFN-1) to induce IFN-stimulated gene (ISG) expression of themselves or indirect antiviral effects by IFN-1 acting on other cells to activate corresponding signaling pathways. In addition, microglia can also exert an antiviral effect by inducing autophagy or secreting cytokines. On the other hand, microglia mediate presynaptic membrane damage in the hippocampus through complement, resulting in long-term memory impairment and cognitive dysfunction in patients with encephalitis. Microglia mediate fetal congenital malformations caused by Zika virus (ZIKV) infection. The gene expression profile of microglia in HIV encephalitis changes, and they tend to be a pro-inflammatory type. Microglia inhibited neuronal autophagy and aggravated the damage of CNS in HIV encephalitis; E3 ubiquitin ligase Pellino (pelia) expressed by microglia promotes the replication of virus in neurons. The interaction between amyloid- $\beta$ peptide (A $\beta$ ) produced by neurons and activated microglia during viral infection is uncertain. Although neurons can mediate antiviral effects by activating receptor-interacting protein kinases 3 (RIPK3) in a death-independent pathway, the RIPK3 pathway of microglia is unknown. Different brain regions have different susceptibility to viruses, and the gene expression of microglia in different brain regions is specific. The relationship between the two needs to be further confirmed. How to properly regulate the function of microglia and make it exert more anti-inflammatory effects is our next research direction.
\end{abstract}

Keywords: Microglia, Viral encephalitis, IFN-1, P2Y12, A $\beta$, Autophagy

\section{Background}

Encephalitis is a common and serious disease, and its clinical manifestations vary from person to person. Its main characteristics include altered mental status and various combinations of acute fever, seizures, neurologic deficits, cerebrospinal fluid (CSF) pleocytosis, and abnormalities in electroencephalographic (EEG) [1]. Although there are many reasons accounting for this disease, the most commonly identified causes are neurotropic viruses. Every year in the USA, there are about seven patients per

\footnotetext{
* Correspondence: 1195273019@qq.com
Department of Neurology, The First Affiliated Hospital, Harbin Medical
University, 23 You Zheng Street, Harbin 150001, Heilong Jiang Province

* Correspondence: 1195273019@qq.com

* Correspondence: 1195273019@qq.com
Department of Neurology, The First Affiliated Hospital, Harbin Medical
University, 23 You Zheng Street, Harbin 150001, Heilong Jiang Province, People's Republic of China
}

100,000 population diagnosed as encephalitis. Among all the cases, approximately half of them have unknown reasons. Of the cases with a known cause, viruses represent $20-50 \%$. Herpes simplex virus (HSV) takes up 50 to $75 \%$ of identified viral cases, with varicella-zoster virus (VZV), enteroviruses, and arboviruses accounting for the majority of the remainders [2]. It is estimated that the median hospitalization charge for a patient with viral encephalitis is $\$ 89,600$ for West Nile virus encephalitis and $\$ 58,000$ for HSV encephalitis. Each year in the USA, approximately 6000 patients are hospitalized for acute viral encephalitis. The total annual cost is up to $\$ 350$ million to $\$ 540$ million, without counting the cost of care after discharge, costs for family caregivers, and lost earning [3]. 
Microglia are CNS-resident mononuclear phagocytic cells characterized by a unique ramified shape and distinctive gene expression [4]. Most microglia are derived from a yolk sac progenitor and seed the brain early in development [5]. As resident cells, microglia are assumed to help orchestrate the immune response to pathogen infection of the brain. While often touted as immune sentinels, little is known about how or if microglia engage in this function [6]. So far, a large body of evidence stemming from both in vitro and in vivo studies have indicated that microglia are capable of responding to viral PAMPs $[7,8]$, but the multifaceted function of microglia involved in viral encephalitis needs further understanding. Recently, how microglia are recruited around infected neurons and the interaction between microglia and other cells has become a hot topic. In this review, we will talk about the function of microglia in viral encephalitis.

\section{Microglia play an anti-inflammatory role in viral encephalitis}

Microglia are recruited around the infected neurons by sensing extracellular ATP signals

Microglia can sense the nucleotides released by infected neurons and are rapidly recruited around infected neurons to exert phagocytic activities in the brain (see Fig. 1). Neurons release more ATP after viral infection, while the levels of ATP, ADP, AMP, and adenosine in cell lysates decrease. Noxious stimuli in neurons trigger a sustained increase in extracellular ATP, which causes microglia to become activated and recruited in minutes to hours [9]. ATP is a potent agonist of the P2Y G protein-coupled receptor [10]. Studies have shown that P2X7 or P2Y12 are both abundant in microglia, and P2Y12 is a microglia-specific marker in the brain [11].

Extracellular ATP is a strong chemotactic signal for microglia. By stimulating the P2Y receptor of microglia,

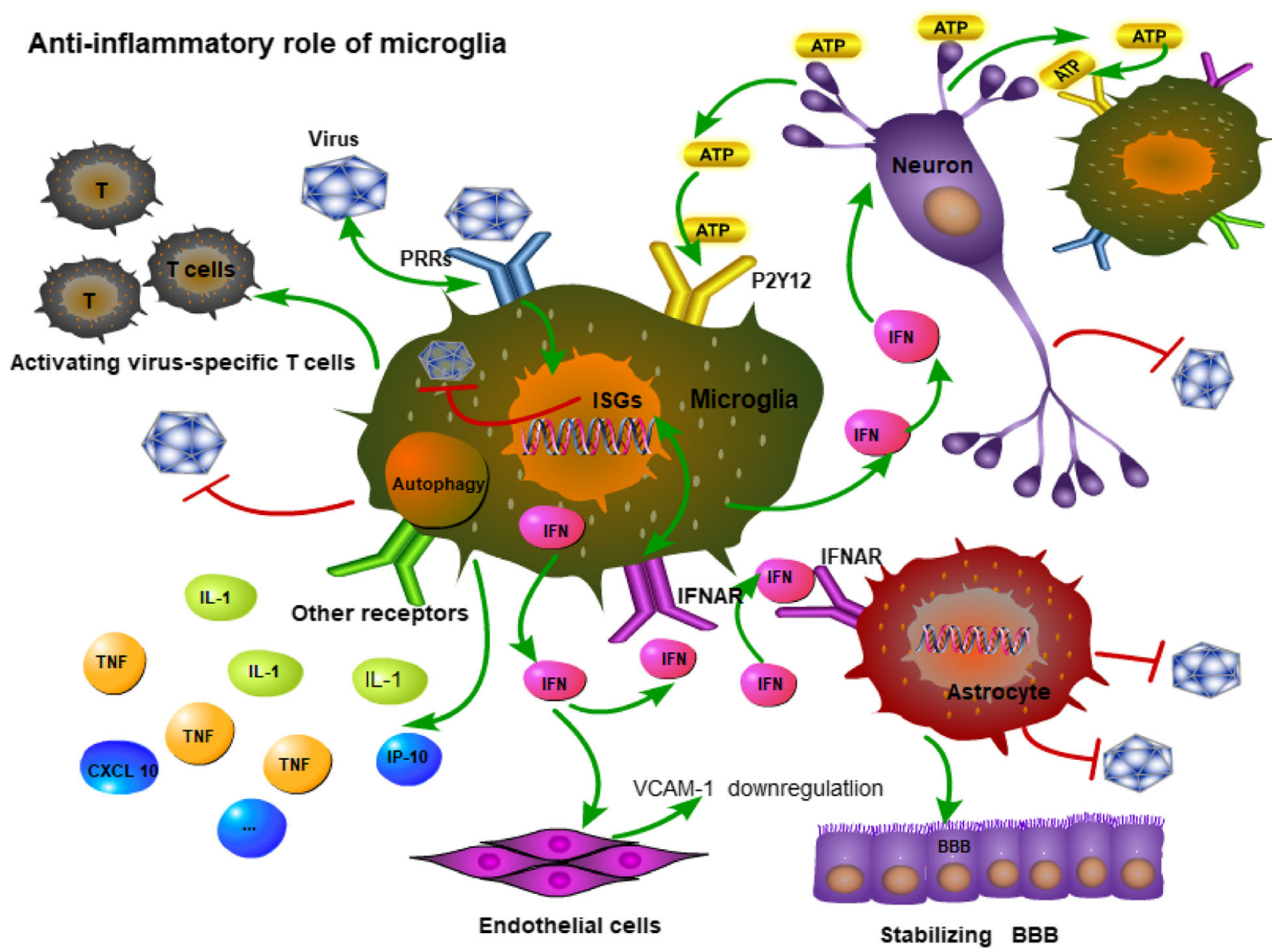

Fig. 1 Illustration of the anti-inflammatory role of microglia in viral encephalitis. The picture shows that microglia can sense ATP signals through the purinergic receptor P2Y12 and are recruited around infected neurons to exert phagocytic activity. Microglia can exert a direct antiviral effect by producing type 1 interferon (IFN-1) to induce IFN-stimulated gene (ISG) expression of themselves. IFN produced by microglia exects indirect antiviral effects by acting on other cells to activate corresponding signaling pathways 
it mediates the rapid response of microglia to the injury site [12]. The number of P2Y12 receptors on the surface of microglia increased over twofold in response to viral infections. Some experiments have built a P2X7- or $\mathrm{P} 2 \mathrm{Y} 12-$ deficient mice model $\left(\mathrm{P}_{\mathrm{XX}} 7^{-/-}\right.$or $\left.\mathrm{P} 2 \mathrm{Y}^{2} 2^{-/-}\right)$in vivo, the lack of $\mathrm{P} 2 \mathrm{X} 7$ has no effect on the recruitment of microglia, whereas an absence of P2Y12 resulted in> $50 \%$ reduction in the numbers of microglia recruited to infected neurons. This indicates that microglia are recruited around the infected neurons via P2Y12 signaling. The CD86+ phagolysosomes of $\mathrm{P} 2 \mathrm{Y}_{12}{ }^{-/-}$microglia decreased significantly compared with normal mice which indicates that P2Y12 is essential for microglia to exert phagocytic activity [9]. Microglia are key contributors to the recruitment of monocytes into the brain during viral infection. In microglia-depleted mice, the phenomenon of monocytes entering the brain is almost completely gone. $\mathrm{CD} 45+$ blood-borne leukocytes were seen in the brain of $\mathrm{P} 2 \mathrm{Y}_{1} 2^{-/-}$mice after infection, and mononuclear cell infiltration was not impaired, which indicates that microglia recruit monocytes through a P2Y12 independent mechanism [9].

Analysis of temporal lobe specimens in patients with herpes simplex type 1 (HSV-1) encephalitis shows that P2Y12-positive microglia processes extend to HSV-1 positive cells, and there are about 1-3 activated microglia around each infected neuron. Microglia appear around the cell bodies and dendrites of neurons before the mature virus particles appear in infected neuronal cells. At this stage, the neuronal membrane is intact and there is a normal chromatin structure in the nucleus. Cell trajectory analysis has shown that the speed of microglia movement in mice with viral encephalitis is reduced, which is related to their tendency to stay around the infected cells [9]. Typical neurological symptoms appear in mice infected with virus when microglia are completely absent, but not P2Y12 deficiency [9]. Consistent with this, mice with microglia depletion have higher viral loads and succumb to infection. The rapidly deteriorating neurological symptoms in microglia-depleted mice may be associated with significantly increased neuronal infection and potential microglial protective mediators such as interleukin-10 deficiency [13].

\section{Microglia play a direct antiviral effect by producing IFN-1 after recognition of virus by pattern recognition receptors (PRRs)}

Mouse hepatitis virus (MHV) is a neurotropic coronavirus. On the fourth day of viral infection, microarray analysis of infected mouse microglia showed that the IFN-1 pathway was the most upregulated [14]. IFN-1 protects the host because treatment with exogenous IFN- $\alpha$ or IFN- $\beta$ limits viral replication, whereas the infection of mice with IFN-1 signaling deficiency can convert nonfatal coronavirus to a lethal type [15]. In the mouse model of vesicular stomatitis virus (VSV) encephalitis, it was found that infected microglia produced IFN-1, and both infected and uninfected microglia upregulated the expression of IFN regulatory factors 7 (IRF7) and activated innate immunity which limits the transsynaptic spread of VSV [16]. Viral infection can induce the expression of IFN-stimulated genes (ISGs), thereby interfering with viral replication and promoting viral clearance [16] (see Fig. 1).

Microglia express a wide range of PRRs, which are important for the defense of HSV-1. Virus induces expression of IFN-1 by the recognition of nucleotides and (RNA-/DNA-sensing) PRRs [7, 8] (see Table 1 for a summary). The combination of IFN-1 and the heterodimeric receptor IFNAR produces a cellular response that initiates a signaling cascade that promotes heterodimers STAT 1/2 nuclear translocation and transcriptional activation of ISGs [17]. The rapid expression of hundreds of ISGs is critical for controlling viral infections because these proteins block the entry, translation, transcription, assembly, and efflux of the virus [18]. Cytosolic dsDNAs can induce a strong innate immune response. GMPAMP synthase (cGAS) is a cytosolic DNA receptor [19]. The binding of cGAS to cytosolic DNA produces a second messenger, cyclic-GMP-AMP (cGAMP), which activates downstream STING and ultimately activates transcription factor IRF3, which promotes IFN- $\beta$ production $[8,20]$.

Different types of PRRs can be involved in the identification of viral infections, including membrane-associated TLRs, cytosolic RNA-sensing RIG-like receptors (RLRs), and DNA sensors. PRRS activates IFN regulatory factors 3 (IRF3) through unique adaptor molecules such as TIR domain-containing adaptor inducing IFN- $\beta$ (TRIF), mitochondrial antiviral signaling (MAVS), and stimulator of type I IFN genes (STING) [21]. Mutations in the IRF3 gene in herpes simplex encephalitis (HSE) patients impaired interferon production and the TLR3-TRIF pathway is the most severely damaged [22]. The cGAS-STING and TLR3-TRIF pathways are the major intrinsic sensing pathways to control HSV1 infection in the CNS. Microglia can induce IFN responses by recognizing viral pathogen-associated molecular patterns (PAMPs) through PRRs. In a typical IFN-1 response pattern, PRRs first induce the production of IFN- $\beta$ after recognition of PAMPs, and then IFN- $\beta$ binds to IFN- $\alpha$ receptor (IFN $\alpha$ R) to initiate IRF7 gene expression in an autocrine or paracrine manner which enables a complete IFN-1 response in viral spread or secondary infections [23]. In order to simulate the natural pathway of HSV-1 entering the central nervous system by retrograde transport, the previous experiment used an ocular infection model. It was confirmed that microglia are the main source of HSV-induced IFN-1, which is induced in a cGAS-STING-dependent manner. Infected cells including 
Table 1 Summary of receptors of microglia involved in viral encephalitis

\begin{tabular}{|c|c|c|c|c|}
\hline Articles & Recognition & Receptors' classification & Location & Effects \\
\hline $\begin{array}{l}\text { Paludan } \\
\text { et al. [19] }\end{array}$ & DNA sensors & TLR3/9; cGAS & $\begin{array}{l}\text { Endosomes; } \\
\text { cytoplasm }\end{array}$ & $\begin{array}{l}\text { Leading to activation of IRFs and induction of IFNsStimulating } \\
\text { autophagy and different types of cell death } \\
\text { Activating the inflammatory NF-kB and inflammasome pathways }\end{array}$ \\
\hline $\begin{array}{l}\text { Mogensen } \\
{[21]}\end{array}$ & $\begin{array}{l}\text { RNA sensors; surface } \\
\text { glycoproteins (GP) } \\
\text { sensors }\end{array}$ & $\begin{array}{l}\text { TLR7/8RLRs (RIG-I, } \\
\text { MDA5); TLR2/4 }\end{array}$ & $\begin{array}{l}\text { Endosome/ } \\
\text { cytoplasm; cell } \\
\text { surface }\end{array}$ & $\begin{array}{l}\text { Resulting in an antiviral response mediated by type I IFN and cytokine } \\
\text { production }\end{array}$ \\
\hline $\begin{array}{l}\text { Sperlagh } \\
\text { et al. [11] }\end{array}$ & $\begin{array}{l}\text { ATP/adenosine } \\
\text { sensors }\end{array}$ & $\begin{array}{l}\mathrm{P} 2 \mathrm{X} 7 / \mathrm{P} 2 \mathrm{X} 4 \mathrm{~A} 1 / \mathrm{A} 2 \mathrm{~A} / \mathrm{A} 3 \\
\text { adenosine receptors }\end{array}$ & Cell surface & $\begin{array}{l}\text { Involved in secretion of various factors, such as } I L-1 \beta \text {, IL-6, and TNF- } \alpha \text {, } \\
\text { mediating apoptosis } \\
\text { Regulating the proliferation/survival of microglia }\end{array}$ \\
\hline $\begin{array}{l}\text { Fekete } \\
\text { et al. [9] }\end{array}$ & ATP & $\mathrm{P} 2 \mathrm{Y} 12$ & Cell surface & $\begin{array}{l}\text { Microglia are recruited around the infected neurons via } \mathrm{P} 2 \mathrm{Y} 12 \text { signaling } \\
\mathrm{P} 2 \mathrm{Y} 12 \text { is essential for microglia to exert phagocytic activity. }\end{array}$ \\
\hline $\begin{array}{l}\text { Vasek } \\
\text { et al. [52] }\end{array}$ & $\begin{array}{l}\text { Complement C3 and } \\
\text { C3 cleavage products }\end{array}$ & C3R & Cell surface & $\begin{array}{l}\text { Damaging of presynaptic ends and resulting in visual-spatial } \\
\text { processing disorders and impaired memory function }\end{array}$ \\
\hline
\end{tabular}

neurons and microglia detected HSV-1 by the cytoplasmic DNA receptor, the adaptor protein STING. The brain of mice lacking cGAS and STING detected higher viral load, and the virus spread widely to the midbrain, hypothalamus, and preoptic area. However, studies have shown that the recruitment of microglia to the infection site is not related to STING [24].

In a model of MHV infection, depletion of microglia using colony-stimulating factor 1 receptor (CSF1R) inhibitors revealed that depletion of microglia at 0-6 days of infection would result in increased mortality and a later depletion had no influences on survival. It indicated that microglia play an important role in controlling viral replication and reducing mortality in the early stage of infection [14]. Upon intranasal vesicular stomatitis virus (VSV) infection, studies have found activated microglia and monocyte aggregation in the olfactory bulb. Studies have also shown that microglia accumulating around the olfactory bulb form a natural immune barrier plays an important role in limiting the spread of VSV in the CNS and preventing lethal encephalitis. The formation of the intrinsic microglia barrier is regulated by IFNAR signaling of neurons and astrocytes and is not regulated by microglia themselves [25]. It has been previously reported that the use of brain-penetrant CSF-1R inhibitor BLZ945 in mice can remove microglia [26]. IL-34, as a CSF-1R ligand, is essential for the maintenance of microglia. BLZ945 plays a role in specifically depleting microglia by inhibiting the interaction of CSF-1R with IL-34 [27]. After depletion of microglia, VSV-infected mice have higher viral load in the brain, cerebellum, and brainstem and have higher mortality, indicating that the formation of microglia barrier in the olfactory bulb is indispensable to limit the spread and spread of VSV [25]. Sall1 is specifically expressed in microglia and is a negative regulator of microglia activation. Sall1 is downregulated in microglia when infected by pathogens, thereby promoting microglia activation [28].

\section{IFN produced by microglia acts on other cells to exert indirect antiviral effect}

Microglia confer STING-dependent antiviral activity to neurons and initiate the production of IFN-1 in astrocytes via the toll-like receptor 3 (TLR3) pathway [24]. Type I IFNAR signaling in astrocytes acts to stabilize the blood-brain barrier $(\mathrm{BBB})$ and protect the brain from viral infections and immunopathology (see Fig. 1). In a mouse model of West Nile virus (WNV) infection, it was found that the permeability of the BBB increased in mice lacking IFNAR, especially in the hindbrain, which leads to an increase in viral entry, indicating that IFNAR signaling has important regulatory effects on BBB permeability in this brain region. The study found that human and mouse cerebellar astrocytes have higher expression of PRRs and ISGs in both steady state and IFN-induced state compared with cerebral cortical astrocytes. Cerebellar astrocytes can reach maximum ISG expression more quickly after receiving IFN stimulation [29]. In vitro studies have shown that IFNAR pathways in brain microvascular endothelial cells (BMECs) and astrocytes can both promote the integrity of tight junction. Studies have shown that IFN-1 stabilizes the integrity of $\mathrm{BBB}$ and promotes the formation of tight junctions by rearranging endothelial junction proteins via Rho-Rac signaling pathways, promoting $\mathrm{BBB}$ tightening and blocking the expression of IL-1 $\beta$ and TNF- $\alpha$ [30].

It is well known that vascular cell adhesion molecule 1 (VCAM-1) is a neuroinflammatory regulator on vascular endothelial cells [31]. IFN-1 regulates immune transport by regulating the expression of VCAM-1 (CD 106) on neurovasculature [32]. IFN- $\beta$ treatment of either human or mouse primary astrocyte cultures from the cerebellum, but not from the cerebral cortex, significantly downregulates VCAM-1 expression [29]. Drug blocking of VCAM-1 binding to its ligand leukocyte adhesion molecule 4 (VLA-4) has been shown to reduce pathogenic neuroinflammation and improve survival after WNV infection [33]. In IFNAR-deficient mice, cerebellar 
infiltrating lymphocytes and macrophages were significantly reduced after treatment with VLA-4 blocker [29]. Loss of astrocyte IFNAR signaling pathway can cause a variety of consequences, including increased expression of inflammatory cytokines and chemokines, which disrupt the blood-brain barrier during neurotropic viral infection [30]. In different brain regions, transcription, immunophenotypic, and bioenergetic properties of microglia are heterogeneous. Cerebellar and hippocampal microglia maintain a stronger immune alert status than microglia in the striatum and cortex. The sensitivity of different brain regions to aging is different, and the cerebellum is the most obvious. This suggests that different brain regions have different responses to aging-related neurodegenerative lesions [34].

Microglia also affect the adaptive immune response. Depletion of microglia changes the response of CD4+ T cells to viral infection in the brain, the total number and percentage of $\mathrm{CD} 4+\mathrm{T}$ cells decrease, and the frequency and number of Tregs also decrease, indicating that microglia are crucial for fully activating virus-specific $\mathrm{T}$ cells. Deletion of microglia in the early stages of WHV infection exacerbates infection, which may be associated with loss of microglial antigen presentation function, as microglia participates in antigen presentation by upregulating Tap1 and Itgax. Depletion of microglia can also result in the loss of major MHC II-expressing cell type, which can also result in decreased expression of MHC II in incoming monocytes/macrophages. Because MHC II is required for re-stimulation and activation of $\mathrm{CD} 4+\mathrm{T}$ cells, a decrease in MHC II reduces the response of virus-specific CD4+ T cells [14].

In addition to producing IFN-1, microglia can also produce other cytokines. The vitro cell culture demonstrates that cytomegalovirus (CMV)-infected astrocytes recruit microglia to the infected foci by releasing monocyte chemoattractant protein 1 (MCP-1). Microglia produces tumor necrosis factor $\alpha(\mathrm{TNF} \alpha)$ and interleukin $1 \beta$ (IL-1 $\beta$ ), interleukin 6 (IL- 6) exerting antiviral effect and inhibiting the replication of CMV in astrocytes [35]. CMV-infected microglia produce $\mathrm{T}$ lymphocyte chemotactic factor CXCL 10, recruiting activated T lymphocytes into the infected area, and T cells produce IFN- $\gamma$ to inhibit virus replication and dissemination [36]. Even with limited viral replication, cytopathic effects were evident in the HSV-infected microglia. Microglia responded to nonpermissive viral infection by producing considerable amounts of TNF- $\alpha$, IL-1 $1 \beta$, and IP- 10 . TNF- $\alpha$ inhibits HSV replication in astrocytes, and IP-10 possesses direct antiviral activity in neurons [37].

\section{Microglia restrict viral infection by autophagy}

Inflammation-induced, STING-dependent autophagy limits Zika virus (ZIKV) infection in Drosophila brains.
The activation of NF-kB dependent on ZIKV induces expression of Drosophila stimulator of interferon genes (dSTING) in the brain. dSTING protects against ZIKV infection by inducing autophagy in the brain. Loss of autophagy leads to increased viral load in the brain and increases infection in Drosophila [38]. Multiple microbial infections activate NFkB-dependent inflammatory signaling cascades, which induce transcription of downstream antimicrobial effectors, including antimicrobial peptides (AMPs), thereby reducing or eliminating infection [39]. Endogenous, bacterial-derived circulating dinucleotides and increased expression of STING mRNA can activate STING [40]. From fruit flies to mammals, activation of antimicrobial autophagy protects cells against a variety of pathogens, including bacteria, viruses, and parasites [41, 42]. Studies have shown that Drosophila infected with ZIKV induces activation of NF-kB signaling in the IMD pathway, thereby activating downstream dSTING. dSTING can limit intracellular pathogens by inducing autophagy [43].

Autophagy protects neurons against a variety of stressors, including viral infections. Autophagy clears infection by capturing pathogens, including viruses, in the cell without causing cell death, which is beneficial for mature neurons $[42,44]$. Previous studies used heat shock-inducible gal4 (HS-gal4) to silence autophagy core genes $\operatorname{Atg} 5$ and $\operatorname{Atg} 7$ in vivo [45] and found that the virus titer of ZIKV in Drosophila is significantly increased. Relish is a transcription factor of NF-kB in the IMD pathway. Using a validated RNAi line which specifically removes Relish from neurons or glial cells in vivo, known as a neuron-specific gal4 driver (elav-gal4) or a glia-specific gal4 driver (Repo-gal4), respectively [46], increased viral infection. In mammals, autophagy limits the replication or pathogenesis of herpes simplex virus type 1 (HSV-1) [47]. Intracellular receptors, such as retinoic acid-inducible gene-I (RIG-I) and melanoma differentiation-associated protein 5 (MDA-5), collectively referred to as RIG-I-like receptors (RLRs), can also detect cytoplasmic viruses nucleic acid [48]. Using the advantages of Drosophila, the study found that the PRR-autophagy axis plays an important role in host defense [49].

\section{Microglia cells play a pro-inflammatory role in viral encephalitis \\ Microglia-mediated synaptic loss causes memory impairment}

Fifty percent of patients with neurotropic WNV infection show chronic cognitive sequelae [50]. Patients who survive after infection with WNV usually present with visual-spatial processing disorders and impaired memory function $[50,51]$. Studies have shown that complement C3 and C3aR mediate the loss of presynaptic ends in mouse hippocampus (see Fig. 2). Mice demonstrate spatial learning in the recovery period of neurological invasive diseases in West Nile. Both neurons and 
microglia express $\mathrm{C} 3 \mathrm{aR}$, which recognizes $\mathrm{C} 3$ cleavage products. $\mathrm{C} 3$ and its cleavage products attract microglia to gather around the neurons to exert phagocytosis activity and to clear the presynaptic ends. In the situation of a neurotropic virus infection, clearance of the presynaptic end may prevent trans-synaptic spread of the virus and stop abnormal signals from infected neurons [52]. Infected and activated microglia in the CNS persist for more than 12 months after receiving antiviral therapy in patients with HSV encephalitis [53]. This may lead to chronic damage to the CNS. Microglia can produce TNF $\alpha[35,36]$. Local increase of TNF $\alpha$ in the hippocampal dentate gyrus activates astrocyte TNF receptor type 1 (TNFR1), which in turn triggers an astrocyte-neuron signaling cascade that results in persistent functional modification of hippocampal excitatory synapses which can lead to cognitive disturbance [54]. Glutamatergic gliotransmission provides a stimulatory input to excitatory synapses in the hippocampal dentate gyrus. TNF $\alpha$ critically controls the process which may influence synaptic transmission and plasticity [55]. This suggests that microglia may also affect synaptic function through cytokines.

\section{Microglia-mediated congenital malformation}

The unprecedented re-emergence of the Zika virus (ZIKV) virus has shocked the world, and reports on microcephaly in Brazil are also increasing [56] (see Fig. 2). In Brazil, the incidence of fetal microcephaly in ZIKV-infected pregnant women is approximately $12.8 \%$ [57]. Surprisingly, there have been increased reports of cases of simultaneous diagnosis of Guillain-Barré syndrome in adults with French Polynesia ZIKV infection [58]. Recent evidence suggests a causal relationship between prenatal ZIKV infection and severe brain abnormalities including microcephaly [59].

ZIKV was detected in the brain of a microcephalic fetus [60]. This means that there is a strong connection between ZIKV and these neurological diseases. The study found that ZIKV mainly infects fetal microglia and induces high levels of pro-inflammatory mediators including IL-6, TNF- $\alpha$, IL-1 $\beta$, and MCP-1. These inflammatory factors may be harmful to the fetus [61]. Microglia's perivascular localization enables them to survey the influx of blood-borne components into the CNS [62]. Thus, microglia are highly susceptible to ZIKV infection. The analysis of vitro culture and microcephalic

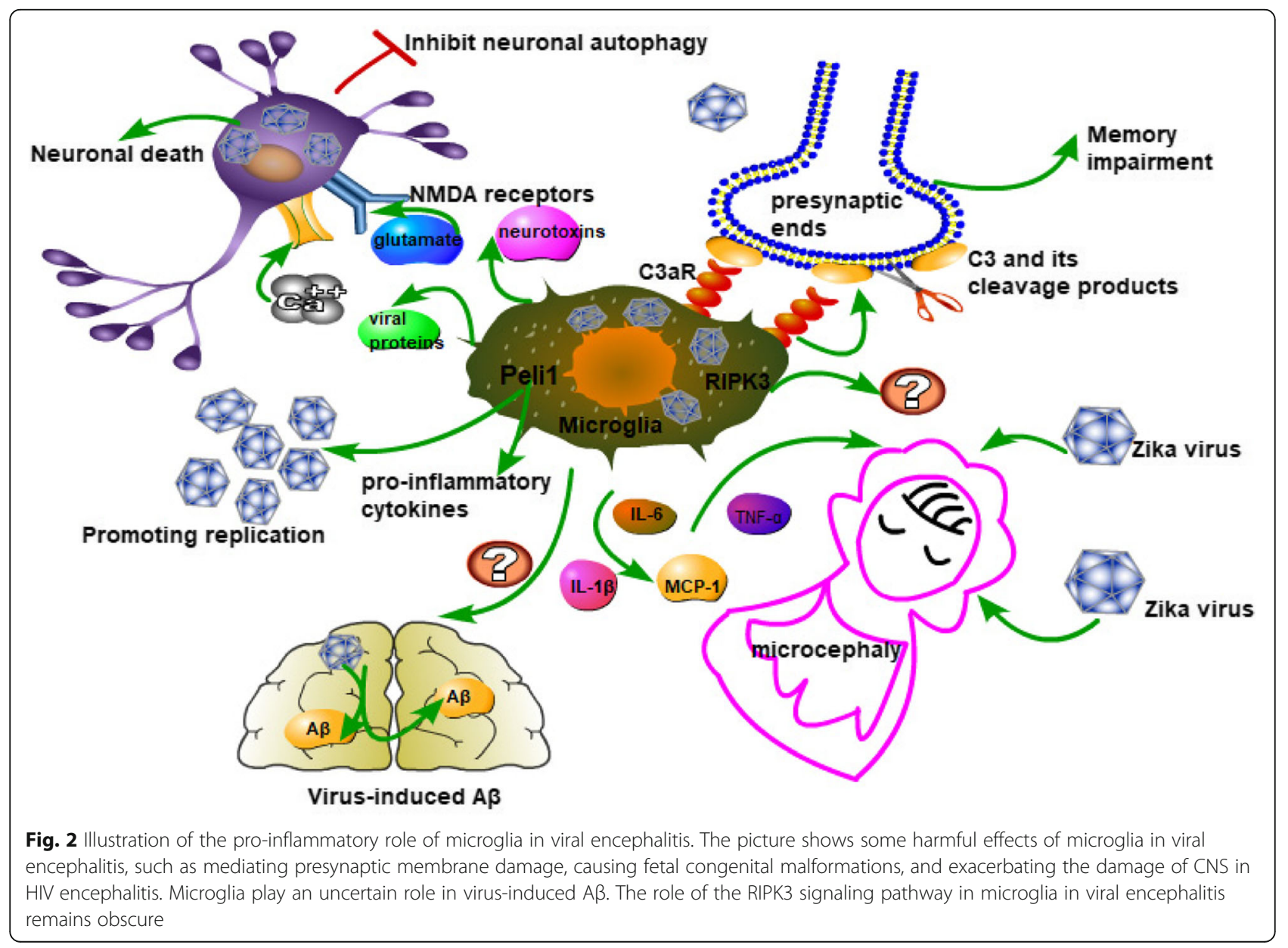


fetal brain histology showed that ZIKV leads to activation of microglia [60]. This, in turn, causes localized neuroinflammation and is accompanied by viral disseminating to the brain parenchyma, which may lead to the development of neuronal death, especially in the cerebral cortex, causing neurological changes and microcephaly. These findings reveal that microglia play an important role in the pathogenesis of congenital ZIKV infection [61].

\section{Microglia mediate neurological damage caused by HIV} Microglia are the main target of HIV-1 infection in the brain [63]. There are viral particles in the infected microglia, suggesting that they may be a potential virus reservoir [64]. The main features of HIV-1 encephalitis are the formation of multinucleated giant cells, microglia nodules, and macrophage infiltration into the central nervous system [65]. The formation of multinucleated giant cells reflects the infection of HIV-1 microglia in vitro. Active infection of microglia is ultimately associated with astrogliosis, myelin pallor, and neuronal loss [65]. Although there are ample evidences confirming that activated microglia have neuropathic effects in HIV-1 encephalitis, there are data suggesting that microglia may have some neuroprotective effects in the early stages of HIV disease [66].

Molecular and histopathology confirm the reduction of autophagy in the brain of patients with HIV-associated dementia. HIV-1-infected microglia release neurotoxins, cytokines, viral proteins, and glutamate; activate NMDA receptors on neurons; cause calcium influx, inhibit neuronal autophagy; enhance apoptotic pathways; and ultimately lead to neuronal death [67]. Enhancing autophagy of neurons in patients with HIV encephalitis may become a target for future treatment. Microarray analyses were performed using brain tissue samples from HIV encephalitis (HIVE) patients, HIV+ patients without encephalitis (HIV/noE), and HIV- controls. It was found that a large number of microglia genes have undergone significant changes during HIV infection, including immune activation and function, kinases, phosphatases, and pro-/anti-apoptotic and neurotrophic factors, indicating that the microglia function is impaired and has a pro-inflammatory tendency [68].

Human cytomegalovirus (HCMV) can cause congenital encephalitis or cause encephalitis in AIDS patients [69]. In patients with advanced AIDS, HCMV-induced central nervous system infection leads to two different neuropathological patterns: microglial nodular encephalitis (MGNE) and ventriculoencephalitis (VE). Microglial nodular encephalitis consists of diffuse microglial cells, aggregated astrocytes, and giant cells. The formation of microglial nodules is considered to be an important cause of HCMV-related dementia in AIDS patients [70].
Recently, a study hypothesized that, in Rasmussen encephalitis (RE), microglial nodules with an upregulation of TLRs provide an environment for the initiation of the later dominating $\mathrm{T}$ cell cytotoxicity. However, in the early stages of viral encephalitis, the interaction between microglial nodules and pathogenic $\mathrm{T}$ cells remains unclear [71].

\section{Microglia and pelia}

In the brain, Peli1 is mainly expressed in microglia. In a mouse model of lethal West Nile virus (WNV) infection, Peli1 promotes the production of pro-inflammatory cytokines and chemokines in microglia and promotes the entry of T cells and macrophages into the CNS. Peli1 plays a crucial role in WNV entry and replication in human and murine neurons and microglia (see Fig. 2). Peli1 promotes adhesion, replication, and synthesis of mature virions. Autopsy of patients who died of WNV encephalitis found that Peli1 was highly expressed on WNV-infected neurons and adjacent inflammatory cells. Peli1-deficient microglia and neurons cause inflammation and reduce host susceptibility to lethal encephalitis [72]. Microglia are the main producers of central nervous system inflammatory cytokines IL- 6 and TNF- $\alpha$ and chemokines CCL2 and CXCL10 after WNV infection [73]. The Peli1-deficient mice have better resistance to $\mathrm{WNV}$, reducing levels of cytokines and chemokines in the body, including IL-6, TNF- $\alpha$, IL- $1 \beta$, and IL-10 and CCL2 and CCL5 [72].

Peli1 expressed in microglia can promote the degradation of TNF receptor-associated factor 3 (Traf3) by regulating TLR/MyD88 signaling and can activate microglia during the induction of experimental autoimmune encephalomyelitis (EAE) [74]. It is well known that microglia respond to viral infection by activating p38MAPK [75]. In WNV-infected Peli1 ${ }^{-1-}$ neurons, phosphorylation levels of p38MAPK and p65 decreased, indicating that Peli1 plays a positive regulatory role in NF- $k B$ and p38MAPK activation [72]. Peli1 facilitates WNV replication in microglia and neurons, especially in the latter, which are the major cells infected during in vivo challenge. It also positively mediates NF- $\mathrm{KB}$ and/or p38MAPK activation in these cells and boosts a robust production of inflammatory cytokines and chemokines, which attracts more infiltration of inflammatory cells from the periphery and ultimately contributes to lethal WNV encephalitis. Thus, Peli1 synergistically promotes virus dissemination and inflammation in the CNS [72].

\section{Microglia play an uncertain role \\ Microglia and virus-induced $A \beta$}

Under physiological conditions, neurons can produce trace amounts of amyloid- $\beta$ peptide $(\mathrm{A} \beta)$ to maintain synaptic plasticity and memory and play an 
antimicrobial role. The clearance of $A \beta$ is achieved by phagocytosis of microglia. HSV reactivation would trigger increased $A \beta$ production, activation of microglia, and release of pro-inflammatory cytokines (pathological state) that would sustain microglia activation and initiate a vicious circle of inflammatory responses. Recurrence of this series of events during life would result in amplification and irreversible damage to hippocampus [76]. Studies have shown that $A \beta$ oligomers can bind glycoproteins on the surface of herpesvirus and accelerate $A \beta$ deposition showing protective viral entrapment activity in 5XFAD mouse and 3D human neural cell culture infection models against neurotropic HSV1. This indicates that $A \beta$ fibrils and deposits may be important in protecting the brain from common viral infections [77]. Carbohydrate-binding promotes $A \beta$ fibrillization and leads to protofibril generation on microbial surfaces. Bound protofibrils first inhibit host cell adhesion by pathogens. Then, propagating $A \beta$ fibrils mediate agglutination and sequestration of microbes within fibrillar $\beta$-amyloid. When the $A \beta$ oligomer-mediated protective antimicrobial pathway is over-activated, progressive $A \beta$ deposition can cause neuroinflammation, neuropathology, and extensive neuronal death, leading to Alzheimer's disease (AD), a model called antimicrobial protection hypothesis [77]. Recently, a 16-year study involving more than 33,000 patients found that HSV 1 infection increased the risk of dementia by 2.56 times. And long-term use of anti-herpes drugs seems to reduce the risk of dementia in patients with HSV-1 infection. In general, patients with shorter ( $<30$ days) or longer ( $\geqq 30$ days) durations of anti-herpetic medications were associated with a decreased risk of dementia, and the treatment duration of $\geqq 30$ days was associated with a lower risk of dementia than those of a duration of $<30$ days [78].

Microglia have been involved in $\mathrm{AD}$ for more than 100 years. Nissl and Alzheimer first described amoebalike glial cells in various neurological states in 1904 and 1910. This amoeba-like glial cell was later confirmed to be a microglia. Microglia were activated in amyloid plaque-enriched areas in Alzheimer's disease brains [79]. Some findings reveal that microglia serve as important physiological functions in learning and memory by promoting learning-related synapse formation through brain-derived neurotrophic factor (BDNF) signaling [80]. In the AD mouse model, a disease-associated microglia (DAM) subtype was found. DAM selectively aggregates around $A \beta$ and exerts phagocytosis. Immunohistochemical staining of mice and human brain slices shows DAM with intracellular/phagocytic $A \beta$ particles [81]. The accumulation of microglia around the $A \beta$ plaque is critical to establishing a physical barrier that limits the spread of plaque and protects surrounding neurons from the toxic effects of the plaque [82].
Interestingly, in a mouse model lacking triggering receptor expressed on myeloid cells 2 (TREM2), microglia could not accumulate around $A \beta$ and the survival and proliferation of microglia around the plaque is impaired. At the same time, the migration of microglia to the lesion is impaired [83, 82]. The microglia lacking TREM2 has metabolic disorders, which are characterized by defects in glycolysis, ATP levels, and anabolism [83]. At the same time, the signaling pathway of TREM 2 is essential for phagocytosis of microglia, and the deletion of TREM 2 leads to aggravated pathology [84]. More malnourished neurons were observed in the brain sections in patients carrying $\mathrm{AD}$-associated TREM2 variants [85]. In contrast, it has been reported that when microglia respond to $A \beta$ plaques, it activates inflammatory bodies, leading to the escalation of inflammation and the release of apoptosis-associated speck-like protein containing a CARD (ASC) specks from inflammasomes, which in turn may initiate the seeding of new plaques [86]. Moreover, excessive activation of microglia in the early stages of $\mathrm{AD}$ may cause excessive synaptic pruning, leading to cognitive impairment [87]. In summary, HSV-1 infection accelerates the deposition of $A \beta$ in the brain and activates microglia. And the role of $A \beta$ during viral encephalitis remains obscure and requires additional study.

\section{Microglia and RIPK3}

Necroptosis is a programmed cell death coordinated by receptor-interacting protein kinases 1 (RIPK1) and 3 (RIPK3). Mixed lineage kinase domain-like protein (MLKL) is an executioner protein that participates in the formation of an apoptotic complex that promotes cell death through cell membrane disruption and cell rupture [88]. Studies have shown that the RIPK3 signaling pathway can control a variety of viral infections, including HSV-1. Murine but not human RIP1/ RIP3 directly senses HSV-1 ICP6 to initiate necroptosis [89]. In the WNV virus infection model, it was found that RIPK 3 inhibits viral pathogenesis by cell death-independent neuroinflammation, mice lacking RIPK 3, or RIPK 1 kinase activity, but not those lacking mlkl or mlkl, and caspase- 8 increased susceptibility to lethal WNV infection. During WNV infection of neurons, RIPK3 is activated in neurons which promotes the expression of chemokines CCL2, CXCL10, etc. and recruits infiltration of immune cells including $\mathrm{T}$ cells, mononuclear macrophages, and antigen presenting cells which exert antiviral effects. This process does not affect the number of microglia in CNS [90]. What role does the RIPK3 signaling pathway in microglia play in viral encephalitis? Further exploration is needed. 


\section{Future perspectives}

Most of the experiments on microglia and viral encephalitis remain in the experimental stage, and many mechanisms need to be further explored before being applied to the clinic. Although microglia can recognize ATP released by infected neurons through P2Y12 and are recruited around neurons, whether there are more other potent chemokines, further exploration is needed. Although IFN-1 plays an antiviral role in most encephalitis, the optimal IFN-1 concentration and optimal application time are still undetermined. Microglia can induce autophagy to exert antiviral effects, but there is insufficient evidence. Microglia produce inflammatory and chemokines and induce adaptive immune responses. Microglia resident in different brain regions have specific gene expression profiles, and different brain regions have different susceptibility to viruses. The relationship between the two remains undetermined, and it is expected that the innate immune response to the virus will be enhanced by changing the gene expression profile of microglia in the future. In WNV infection, microglia mediate presynaptic terminal loss in the hippocampus though recognizing $\mathrm{C} 3$ by $\mathrm{C} 3 \mathrm{R}, \mathrm{C} 3$, or C3R-specific antibodies may block this process. Microglia inhibit autophagy in patients with HIVE, aggravating CNS injury. How to inhibit or delete the function of microglia or how to enhance the autophagy of neurons will become the direction of future research. There is a strong relationship between fetal cerebellar malformation and activation of microglia in ZIKV infection, and it is necessary to develop microglia inhibitors that are safe for pregnant women and fetuses. Pelia of microglia promotes viral replication in neurons, and moderate blockade of pelia may become a target for future treatment. In viral encephalitis, $A \beta$ produced by neurons increases the risk of dementia in patients. Studies show that prolonging antiviral time will reduce the risk of dementia. The interaction between $A \beta$ and activated microglia in patients with viral encephalitis is unknown. It is unknown whether moderately enhancing the phagocytosis of microglia can reduce the risk of dementia. It needs further research. Traditionally, activation of RIPK3 will result in the formation of an apoptotic complex that mediates cell death. Recent studies have shown that in viral encephalitis, activation of neuronal RIPK3 exerts an antiviral effect in a death-independent pathway, and the RIPK3 pathway of microglia requires further investigation.

\section{Conclusions}

Microglia account for $10 \%$ of the total number of cells in the adult central nervous system [91]. In a healthy CNS, microglia are not "resting" but in a highly active "surveillance and rapid response" state [92]. Microglia processes are dynamic, continuously scanning the environment and sampling it [93]. They are able to detect changes in $\mathrm{pH}$, purines, cytokines, chemokines, amino acids, and inorganic compounds [94]. Microglia are derived from the primitive macrophages of the yolk sac, which migrate to the developing central nervous system. They have the ability to self-renew, thus maintaining their number throughout life without any input from the bone marrow-derived precursor cells [95, 96]. Microglia can respond to neurons, which in turn affects neuronal activity. Microglia are involved in the programmed death of neurons and the apoptosis and clearance of new neurons during development. The activity of microglia promotes the pruning, elimination, and maturation of synapses [92]. Microglia serve important physiological functions in learning and memory by promoting learning-related synapse formation through BDNF signaling [80]. The phagocytic activity of microglia is essential for the clearance of senescent cells and debris [97], slowing the toxic effects of amyloid- $\beta$ [98]. Microglia are not only important cells that maintain the homeostasis of CNS, but also respond to injury, infection, and neurodegeneration through proliferation and altered transcription and morphology [99, 92]. Microglia are often touted as the first responder to CNS infection and respond quickly to injury [93]. However, depending on the stimulus received, this activation profile is also different and may result in harmful or beneficial effects $[100,6]$. HSV-1 can be treated, but can WNV or enteroviruses? The high morbidity and high morbidity rate of viral encephalitis have caused widespread concern. Although traditional antiviral therapy is effective, there are still many shortcomings. Research on microglia and viral encephalitis provides new targets for treatment. Microglia play a unique role in different encephalitis. The current research on microglia and viral encephalitis remains at the experimental stage. In the future, depending on the type of encephalitis, rationally enhancing or inhibiting the activation of microglia will greatly improve the therapeutic effect of encephalitis, improve the prognosis of traditional antiviral therapy, and reduce the sequelae of encephalitis.

\footnotetext{
Abbreviations

AD: Alzheimer's disease; AIDS: Human acquired immunodeficiency syndrome; AMPs: Antimicrobial peptides; ASC: Apoptosis-associated speck-like protein containing a CARD; A $\beta$ : Amyloid- $\beta$ peptide; BBB: Blood-brain barrier; BDNF: Brain-derived neurotrophic factor; BMECs: Brain microvascular endothelial cells; CGAMP: Cyclic-GMP-AMP; cGAS: GMP-AMP synthase; CMV: Cytomegalovirus; CNS: Central nervous system; CSF: Cerebrospinal fluid; CSF1R: Colony-stimulating factor 1 receptor; DAM: Disease-associated microglia; dSTING: Drosophila stimulator of interferon genes; EAE: Experimental autoimmune encephalomyelitis;

EEG: Electroencephalographic; elav-gal4: A neuron-specific gal4 driver; HCMV: Human cytomegalovirus; HIV/noE: HIV+ patients without encephalitis; HIVE: HIV encephalitis; HSE: Herpes simplex encephalitis; HS-gal4: Heat shock-
} 
inducible gal4; HSV: Herpes simplex virus; IFN-1: Type 1 interferon; IFNa: Interferon a; IFNaR: IFN-a receptor; IFNaR: Interferon receptor; IFN$\beta$ : Interferon $\beta$; IL-6: Interleukin 6; IL-1 $\beta$ : Interleukin 1 $\beta$; IL-34: Interleukin 34; IP-10: IFN- $\gamma$ inducible protein $10 \mathrm{kD}$; IRF3: IFN regulatory factors 3; IRF7: IFN regulatory factors 7; ISGs: IFN-stimulated genes; MAVS: Mitochondrial antiviral signaling; MCP-1: Monocyte chemoattractant protein 1; MDA-5: Melanoma differentiation-associated protein 5; MGNE: Microglial nodular encephalitis; MHV: Mouse hepatitis virus; MLKL: Mixed lineage kinase domain-like protein; NMDA: N-methyl-D-aspartic acid receptor; P2Y12 $2^{-1-}$ : P2Y12 deficiency; PAMPs: Pathogen-associated molecular patterns; Peli1: E3 ubiquitin ligase Pellino 1; PRR: Pattern recognition receptors; RE: Rasmussen encephalitis; Repo-gal4: A glia-specific gal4 driver; RIG-I: Retinoic acid-inducible gene-1; RIPK1: Receptor-interacting protein kinases 1; RIPK3: Receptor-interacting protein kinases 3; RLRs: RNA-sensing RIG-like receptors; STAT 1/2: Signal transducer and activator of transcription 1/2; STING: Stimulator of type I IFN genes; TLR3: Toll-like receptor 3; TNFR1: TNF receptor type 1; TNF-a: Tumor necrosis factor a; Traf3: TNF receptor-associated factor 3; TREM2: Triggering receptor expressed on myeloid cells 2; TRIF: TIR domain-containing adaptor inducing IFN- $\beta$; VCAM-1: Vascular cell adhesion molecule 1;

VE: Ventriculoencephalitis; VLA-4: Leukocyte adhesion molecule; VSV: Vesicular stomatitis virus; VZV: Varicella-zoster virus; WNV: West Nile virus; ZIKV: Zika virus

\section{Acknowledgements \\ Not applicable.}

\section{Funding}

Not applicable.

\section{Availability of data and materials}

Authors confirm that all relevant data are included in the article.

\section{Authors' contributions}

ZC carried out the literature review, participated in the sequence alignment, and drafted the manuscript. DZ helped to draft the manuscript. GL conceived, designed, and coordinated the study and contributed to and finalized the draft. All authors read and approved the final manuscript.

\section{Ethics approval and consent to participate}

Not applicable.

\section{Consent for publication}

Not applicable as no patients/participants involved in this review.

\section{Competing interests}

The authors declare that they have no competing interests.

\section{Publisher's note}

Springer Nature remains neutral with regard to jurisdictional claims in published maps and institutional affiliations.

\section{Received: 22 November 2018 Accepted: 24 February 2019} Published online: 09 April 2019

\section{References}

1. Tyler KL. Acute Viral Encephalitis Kenneth L.N Engl J Med. 2018:379:557-66.

2. George BP, Schneider EB, Venkatesan A. Encephalitis hospitalization rates and inpatient mortality in the United States, 2000-2010. PLoS One. 2014;9:e104169.

3. Vora NM, Holman RC, Mehal JM, Steiner CA, Blanton J, Sejvar J. Burden of encephalitis-associated hospitalizations in the United States, 1998-2010. Neurology. 2014;82:443-51.

4. Hickman SE, et al. The microglial sensome revealed by direct RNA sequencing. Nat Neurosci. 2013;16:1896-19.

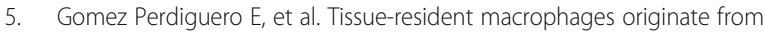
yolk-sac-derived erythro-myeloid progenitors. NaSture. 2015;518:547-51.

6. Crotti A, Ransohoff RM. Microglial physiology and pathophysiology: insights from genome-wide transcriptional profiling. Immunity. 2016:44:505-15.

7. Goubau D, Deddouche S, Reis e Sousa C. Cytosolic sensing of viruses. Immunity. 2013;38:855-869.
8. Paludan SR. Activation and regulation of DNA-driven immune responses. Microbiol Mol Biol Rev. 2015;79:225-41.

9. Fekete R, Cserép C, Lénárt N, Tóth K, Orsolits B, Martinecz B, et al. Microglia control the spread of neurotropic virus infection via P2Y12 signalling and recruit monocytes through $\mathrm{P} 2 \mathrm{Y} 12$-independent mechanisms. Acta Neuropathol. 2018;136:461-82.

10. Illes $\mathrm{P}$, Alexandre RJ. Molecular physiology of $\mathrm{P} 2$ receptors in the central nervous system. Eur J Pharmacol. 2004;483:5-17.

11. Sperlagh B, Illes P. Purinergic modulation of microglial cell activation. Purinergic Signal. 2007;3:117-27.

12. Davalos D, Grut zendler J, Yang G, Kim JV, Zuo Y, Jung S, Littman DR, Dustin ML, Gan WB. ATP mediates rapid microglial response to local brain injury in vivo. Nat Neurosci2005; 8:752-758.

13. Garcia JM, Stillings SA, Leclerc JL, Phillips H, Edwards NJ, Robicsek SA, Hoh BL, Blackburn S. Dore S role of interleukin-10 in acute brain injuries. Front Neurol. 2017;8:244

14. Wheeler DL, Sariol A, Meyerholz DK, Perlman S. Microglia are required for protection against lethal coronavirus encephalitis in mice. J Clin Invest. 2018:128:931-43.

15. Cervantes-Barragan $L$, et al. Control of coronavirus infection through plasmacytoid dendritic-cellderived type I interferon. Blood. 2007;109:1131-7.

16. Drokhlyansky E, Goz Ayturk D, Soh TK, Chrenek R, O'Loughlin E, Madore C, Butovsky O, Cepko CL. The brain parenchyma has a type I interferon response that can limit virus spread. Proc Natl Acad Sci USA. 2017;114:E95-E104.

17. Platanias LC. Mechanisms of type-l- and type-II-interferon-mediated signalling. Nat Rev Immunol. 2005;5:375-86.

18. Lazear HM, Diamond MS. New insights into innate immune restriction of West Nile virus infection. Curr Opin Virol. 2015;11:1-6.

19. Paludan SR, Bowie AG. Immune sensing of DNA. Immunity. 2013;38:870-80.

20. Sun L, Wu J, Du F, Chen X, Chen ZJ. Cyclic GMP-AMP synthase is a cytosolic DNA sensor that activates the type I interferon pathway. Science. 2013;339:786-91.

21. Mogensen TH. Pathogen recognition and inflammatory signaling in innate immune defenses. Clin Microbiol Rev. 2009;22:240-73.

22. Andersen $\mathrm{LL}$, et al. Functional IRF3 deficiency in a patient with herpes simplex encephalitis. J Exp Med. 2015;212:1371-9.

23. Perry AK, Chen G, Zheng D, Tang H, Cheng G. The host type I interferon response to viral and bacterial infections. Cell Res. 2005;15:407-22.

24. Reinert LS, Lopusna K, Winther H, Sun C, Thomsen MK, Nandakumar R, Mogensen TH, Meyer M, Vaegter C, Nyengaard JR, et al. (2016) Sensing of HSV-1 by the CGAS-STING pathway in microglia orchestrates antiviral defence in the CNS. Nat Commun. 2016;7:13348.

25. Chhatbar C, Detje CN, Grabski E, Borst K, Spanier J, Ghita L, Elliott DA, et al. Type I interferon receptor signaling of neurons and astrocytes regulates microglia activation during viral encephalitis. Cell Rep. 2018;25:118-29.

26. Pyonteck SM, Akkari L, Schuhmacher AJ, Bowman RL, Sevenich L, Quail DF, Olson OC, Quick ML, Huse JT, Teijeiro V, et al. CSF-1R inhibition alters macrophage polarization and blocks glioma progression. Nat Med. 2013;19:1264-72.

27. Greter M, Lelios I, Pelczar P, Hoeffel G, Price J, Leboeuf M, Kündig TM, Frei K, Ginhoux F, Merad M, Becher B. Stroma-derived interleukin-34 controls the development and maintenance of langerhans cells and the maintenance of microglia. Immunity. 2012;37:1050-60.

28. Buttgereit A, Lelios I, Yu X, Vrohlings M, Krakoski NR, Gautier EL, Nishinakamura R, Becher B, Greter M. Sall1 is a transcriptional regulator defining microglia identity and function. Nat Immunol. 2016;17:1397-406.

29. Daniels BP, Jujjavarapu H, Durrant DM, Williams JL, Green RR, White JP, Lazear HM, Gale M Jr, Diamond MS, Klein RS. Regional astrocyte IFN signaling restricts pathogenesis during neurotropic viral infection. J Clin Invest. 2017;127:843-56.

30. Daniels BP, Holman DW, Cruz-Orengo L, Jujjavarapu H, Durrant DM, Klein RS Viral pathogen-associated molecular patterns regulate blood-brain barrier integrity via competing innate cytokine signals. MBio. 2014;5:e01476-14.

31. Engelhardt B, Ransohoff RM. Capture, crawl, cross: the T cell code to breach the blood-brain barriers. Trends Immunol. 2012;33:579-89.

32. Bitsch $A$, et al. Interferon beta-1b modulates serum SVCAM-1 levels in primary progressive multiple sclerosis. Acta Neurol Scand. 2004;110:386-92.

33. Getts DR, Terry RL, Getts MT, Muller M, Rana S, Deffrasnes C, Ashhurst TM, Radford J, Hofer M, Thomas S, Campbell IL, King NJ. Targeted blockade in lethal West Nile virus encephalitis indicates a crucial role for very late 
antigen (VLA)-4-dependent recruitment of nitric oxide-producing macrophages.J Neuroinflammation. 2012;9:246.

34. Grabert $\mathrm{K}$, et al. Microglial brain region-dependent diversity and selective regional sensitivities to aging. Nat Neurosci. 2016;19:504-16.

35. Cheeran MC, Hu S, Yager SL, Gekker G, Peterson PK, Lokensgard JR. Cytomegalovirus induces cytokine and chemokine production differentially in microglia and astrocytes: antiviral implications. J NeuroOncol. 2001;7:135-47

36. Cheeran MC, Hu S, Sheng WS, Peterson PK, Lokensgard JR. CXCL10 production from cytomegalovirus-stimulated microglia is regulated by both human and viral interleukin-10. J Virol. 2003;77:4502-15.

37. Lokensgard JR, Hu S, Sheng W, vanOijen M, cox D, Cheeran MC, Peterson PK. Robust expression of TNF-alpha, IL-1 beta, RANTES, and IP-10 by human microglial cells during nonproductive infection with herpes simplex virus. J Neuro-Oncol 2001;7:208-219.

38. Liu Y, Gordesky-Gold B, Leney-Greene M, Weinbren NL, Tudor M, Cherry S. Inflammation-induced, STING-dependent autophagy restricts Zika virus infection in the Drosophila brain. Cell Host Microbe. 2018;24:57-68.

39. Buchon N, Silverman N, Cherry S. Immunity in Drosophila melanogasterfrom microbial recognition to whole-organism physiology. Nat Rev Immunol. 2014:14:796-810.

40. Burdette DL, Vance RE. STING and the innate immune response to nucleic acids in the cytosol. Nat Immunol. 2013;14:19-26.

41. Choi J, Park S, Biering SB, Selleck E, Liu CY, Zhang X, Fujita N, Saitoh T, Akira $S$, Yoshimori $T$, et al. The parasitophorous vacuole membrane of toxoplasma gondii is targeted for disruption by ubiquitin-like conjugation systems of autophagy. Immunity. 2014:40:924-35.

42. Moy RH, Gold B, Molleston JM, Schad V, Yanger K, Salzano MV, Yagi Y, Fitzgerald KA, Stanger BZ, Soldan SS, Cherry S. Antiviral autophagy restricts Rift Valley fever virus infection and is conserved from flies to mammals. Immunity. 2014;40:51-65.

43. Moretti J, Roy S, Bozec D, Martinez J, Chapman JR, Ueberheide B, Lamming DW, Chen ZJ, Horng T, Yeretssian G, et al. STING senses microbial viability to orchestrate stress-mediated autophagy of the endoplasmic reticulum. Cell. 2017; 171:809-23

44. Levine B, Mizushima N, Virgin HW. Autophagy in immunity and inflammation. Nature. 2011;469:323-35.

45. Scott RC, Schuldiner O, Neufeld TP. Role and regulation starvation-induced autophagy in the Drosophila fat body. Dev Cell. 2014:167-78.

46. Morris O, Liu X, Domingues C, Runchel C, Chai A, Basith S, Tenev T, Chen H, Choi S, Pennetta G, et al. Signal integration by the IkB protein pickle shapes Drosophila innate host defense. Cell Host Microbe. 2016;20:283-95.

47. Joubert PE, Werneke SW, de la Calle C, Guivel-Benhassine F, Giodini A, Peduto L, Levine B, Schwartz O, Lenschow DJ, Albert ML. Chikungunya virus-induced autophagy delays caspase-dependent cell death. J Exp Med. 2012;209:1029-47.

48. Loo YM, Gale M. Immune signaling by RIG--like receptors. Immunity. 2011;34:680-92

49. Nakamoto M, Moy RH, Xu J, Bambina S, Yasunaga A, Shelly SS, Gold B, Cherry S. Virus recognition by Toll-7 activates antiviral autophagy in Drosophila. Immunity. 2012;36:658-67.

50. Sejvar JJ, et al. Neurologic manifestations and outcome of West Nile virus infection. J Am Med Assoc. 2003;290:511-5.

51. Sadek JR, Pergam SA, Harrington JA, Echevarria LA, Davis LE, Goade D, Harnar J, Nofchissey RA, et al. Persistent neuropsychological impairment associated with West Nile virus infection. J Clin Exp Neuropsychol. 2010;32:81-7.

52. Vasek MJ, Garber C, Dorsey D, Durrant DM, Bollman B, Soung A, Yu J, Perez-Torres C, Frouin A, Wilton DK, et al. A complement-microglial axis drives synapse loss during virus-induced memory impairment. Nature. 2016;534:538-43.

53. Cagnin AR, Myers RN, Gunn AD, Lawrence T, Stevens GW, Kreutzberg T, et al. In vivo visualization of activated glia by [11C] (R)-PK11195-PET following herpes encephalitis reveals projected neuronal damage beyond the primary focal lesion. Brain. 2001:124:2014-27.

54. Habbas S, Santello M, Becker D, Stubbe H, Zappia G, Liaudet N, Klaus FR, Kollias G, Fontana A, Pryce CR, Suter T, Volterra A, Neuroinflammatory TNFa impairs memory via astrocyte signaling. Cell;2015;163:1730-41.

55. Santello M, Bezzi $P$, Volterra A. TNFa controls glutamatergic gliotransmission in the hippocampal dentate gyrus. Neuron. 2011;69:988-1001.

56. Brasil P, Pereira JP Jr, Raja Gabaglia C, et al. Zika virus infection in pregnant women in Rio de Janeiro. N Engl J Med. 2016;375:2321-34.
57. Moron AF, Cavalheiro S, Milani H, et al. Microcephaly associated with maternal Zika virus infection. BJOG. 2016;123:1265-9.

58. Cao-Lormeau VM, Blake A, Mons S, et al. Guillain-Barré syndrome outbreak associated with Zika virus infection in French Polynesia: a case-control study. Lancet. 2016;387:1531-9.

59. Rasmussen SA, Jamieson DJ, Honein MA, Petersen LR. Zika virus and birth defects-reviewing the evidence for causality. N Engl J Med. 2016;374:1981-7.

60. Mlakar J, Korva M, Tul N, et al. Zika virus associated with microcephaly. N Engl J Med. 2016;374:951-8.

61. Lum FM, Low DK, Fan Y, Tan JJ, Lee B, Chan JK, Rénia L, Ginhoux F, Ng LF Zika virus infects human fetal brain microglia and induces inflammation. Clin Infect Dis. 2017;64:914-20.

62. Dudvarski Stankovic N, Teodorczyk M, Ploen R, Zipp F, Schmidt MHH. Microglia-blood vessel interactions: a double-edged sword in brain pathologies. Acta Neuropathol. 2016;131:347-63.

63. Cosenza MA, Zhao ML, Si Q, Lee SC. Human brain parenchymal microglia express CD14 and CD45 and are productively infected by HIV-1 in HIV-1 encephalitis. Brain Pathol. 2002;12:442-55.

64. Williams KC, Hickey WF. Central nervous system damage, monocytes and macrophages, and neurological disorders in AIDS. Annu Rev Neurosci. 2002;25:537-62.

65. Persidsky Y, Gendelman HE. Mononuclear phagocyte immunity and the neuropathogenesis of HIV-1 infection. J Leukoc Biol. 2003;74:691-701.

66. Gras GF, Chretien AV, Vallat-Decouvelaere G, Le Pavec F, Porcheray C, Bossuet C, Leone P, Mialocq N, Dereuddre-Bosquet P, et al. Regulated expression of sodium-dependent glutamate transporters and synthetase: a neuroprotective role for activated microglia and macrophages in HIV infection? Brain Pathol. 2003:13:211-22.

67. Alirezaei M, Kiosses WB, Fox HS. Decreased neuronal autophagy in HIV dementia: a mechanism of indirect neurotoxicity. Autophagy. 2008:4:963-6.

68. Ginsberg SD, Alldred MJ, Gunnam SM, Schiroli C, Lee SH, Morgello S, Fischer T. Expression profiling suggests microglial impairment in HIV neuropathogenesis. Ann Neurol. 2018;83:406-17.

69. Arribas JR, Storch GA, Clifford DB, Tselis AC. Cytomegalovirus encephalitis. Annl ntern Med. 1996;125:577-87.

70. Grassi MP, Clerici F, Perin C, D'Arminio Monforte A, Vago L, Borella M, Boldorini R, Mangoni A. Microglial nodular encephalitis and ventriculoencephalitis due to cytomegalovirus in patients with AIDS: two distinct clinical patterns. Clin Infect Dis. 1998;27:504-8.

71. Tröscher AR, Wimmer I, emada-Garrido L, Köck U. Gessl D, et al. Acta Neuropathol: Microglial nodules provide the environment for pathogenic T cells in human encephalitis; 2019.

72. Luo H, Winkelmann ER, Zhu S, Ru W, Mays E, Silvas JA, Vollmer LL, et al. Peli1 facilitates virus replication and promotes neuroinflammation during West Nile virus infection. J Clin Invest. 2018:128:4980-91.

73. Cheeran MC, Hu S, Sheng WS, Rashid A, Peterson PK, Lokensgard JR. Differential responses of human brain cells to West Nile virus infection. $J$ Neuro-Oncol. 2005;11:512-5

74. Xiao Y, Jin J, Chang M, Chang JH, Hu H, Zhou X, Brittain GC, Stansberg C, et al. Peli1 promotes microglia-mediated CNS inflammation by regulating Traf3 degradation. Nat Med. 2013;19:595-602.

75. Town T, Jeng D, Alexopoulou L, Tan J, Flavell RA. Microglia recognize double-stranded RNA via TLR3. J Immunol. 2006;176:3804-12.

76. Bourgade K, Le Page A, Bocti C, Witkowski JM, Dupuis G, Frost EH, Fulop T. Protective effect of amyloid- $\beta$ peptides against herpes simplex Virus-1 infection in a neuronal cell culture model. J Alzheimers Dis. 2016;50:1227-41.

77. Eimer WA, Vijaya Kumar DK, Navalpur Shanmugam NK, Rodriguez AS, Mitchell T, Washicosky KJ, György B, Breakefield XO, Tanzi RE, Moir RD. Alzheimer's disease-associated $\beta$-amyloid is rapidly seeded by herpesviridae to protect against brain infection. Neuron. 2018;99:56-63.

78. Tzeng NS, Chung CH, Lin FH, Chiang CP, Yeh CB, Huang SY, Lu RB, Chang HA, Kao YC, Yeh HW, et al. Anti-herpetic medications and reduced risk of dementia in patients with herpes simplex virus infections-a Nationwide. Population-Based Cohort Study in Taiwan Neurotherapeutics. 2018;15:417-29.

79. Lue LF, Kuo YM, Beach T, Walker DG. Microglia activation and antiinflammatory regulation in Alzheimer's disease. Mol Neurobiol. 2010:41:115-28.

80. Parkhurst CN, Yang G, Ninan I, Savas JN, et al. Microglia promote learning dependent synapse formation through brain-derived neurotrophic factor. Cell. 2013;155:1596-609. 
81. Keren-Shaul H, Spinrad A, Weiner A, Matcovitch-Natan O, Dvir-Szternfeld R, Ulland TK, David E, et al. A unique microglia type associated with restricting development of Alzheimer's disease. Cell. 2017;169:1276-90.

82. Mazaheri F, Snaidero N, Kleinberger G, Madore C, Daria A, et al. TREM2 deficiency impairs chemotaxis and microglial responses to neuronal injury. EMBO Rep. 2017;18:1186-98.

83. Ulland TK, Song WM, Huang SC, Ulrich JD, Sergushichev A, Beatty WL, Loboda AA, et al. TREM2 maintains microglial metabolic fitness in Alzheimer's disease. Cell. 2017;170:649-63.

84. Wang Y, Ulland TK, Ulrich JD, Song W, Tzaferis JA, Hole JT, Yuan P, Mahan TE, Shi Y, Gilfillan S, et al. TREM2-mediated early microglial response limits diffusion and toxicity of amyloid plaques. J Exp Med. 2016b;213:667-75.

85. Yuan P, Condello C, Keene CD, Wang Y, Bird TD, Paul SM, Luo W, Colonna M, Baddeley D, Grutzendler J. TREM2 haplodeficiency in mice and humans impairs the microglia barrier function leading to decreased amyloid compaction and severe axonal dystrophy. Neuron. 2016;90:724-39.

86. Venegas C, Kumar S, Franklin BS, Dierkes T, Brinkschulte R, Tejera D, et al. Microglia-derived ASC specks cross seed amyloid-beta in Alzheimer's disease. Nature. 2017;552:355-61.

87. Hong S, Beja-Glasser VF, Nfonoyim BM, Frouin A, Li S, et al. Complement and microglia mediate early synapse loss in Alzheimer mouse models. Science. 2016:352:712-6.

88. Wang H, Sun L, Su L, Rizo J, Liu L, Wang LF, Wang FS, Wang X. Mixed lineage kinase domain-like protein MLKL causes necrotic membrane disruption upon phosphorylation by RIP3. Mol Cell. 2014;54:133-46.

89. Huang Z, Wu SQ, Liang Y, Zhou X, Chen W, Li L, Wu J, Zhuang Q, Chen C, Li J, Zhong CQ, Xia W, Zhou R, Zheng C, Han J. RIP1/RIP3 binding to HSV-1 ICP6 initiates necroptosis to restrict virus propagation in mice. Cell Host Microbe. 2015;17:229-42.

90. Daniels BP, Snyder AG, Olsen TM, Orozco S, Oguin TH, Tait SWG, Martinez J, Gale M, LooYM, Oberst a. RIPK3 restricts viral pathogenesis via cell death independent neuroinflammation.Cell.2017;169:301-313.

91. Aguzzi A, Barres BA, Bennett ML. Microglia: scapegoat, saboteur, or something else? Science. 2013;339:156-61.

92. Salter MW, Beggs S. Sublime microglia: expanding roles for the guardians of the CNS. Cell. 2014;158:15-24.

93. Nimmerjahn A, Kirchhoff F, Helmchen F. Resting microglial cells are highly dynamic surveillants of brain parenchyma in vivo. Science. 2005;308:1314-8.

94. Prinz M, Priller J, Sisodia SS, Ransohoff RM. Heterogeneity of CNS myeloid cells and their roles in neurodegeneration. Nat Neurosci. 2011;14:1227-35.

95. Greter M, Merad M. Regulation of microglia development and homeostasis. Glia. 2013;61:121-7.

96. Gomez Perdiguero E, Schulz C, Geissmann F. Development and homeostasis of "resident" myeloid cells: the case of the microglia. Glia. 2013;61:112-20.

97. Takahashi K, Rochford CD, Neumann H. Clearance of apoptotic neurons without inflammation by microglial triggering receptor expressed on myeloid cells-2. J Exp Med. 2005;201:647-57.

98. Farfara D, Trudler D, Segev-Amzaleg N, Galron R, Stein R, Frenkel D. Gsecretase component presenilin is important for microglia b-amyloid clearance. Ann Neurol. 2011;69:170-80.

99. Herz J, Filiano AJ, Smith A, Yogev N, Kipnis J. Myeloid cells in the central nervous system. Immunity. 2017;46:943-56.

100. Yamasaki R, et al. Differential roles of microglia and monocytes in the inflamed central nervous system. J Exp Med. 2014;211:1533-49.

Ready to submit your research? Choose BMC and benefit from:

- fast, convenient online submission

- thorough peer review by experienced researchers in your field

- rapid publication on acceptance

- support for research data, including large and complex data types

- gold Open Access which fosters wider collaboration and increased citations

- maximum visibility for your research: over $100 \mathrm{M}$ website views per year

At BMC, research is always in progress.

Learn more biomedcentral.com/submissions 